\title{
Novel long noncoding RNA GACAT3 promotes colorectal cancer cell proliferation, invasion, and migration through miR-I49
}

This article was published in the following Dove Press journal:

OncoTargets and Therapy

\author{
Weiwei Zhou' \\ Lei Wang ${ }^{2}$ \\ Yu Miao' \\ Rongge Xing' \\ 'Department of Pathology, \\ Central Hospital of Cangzhou, \\ Hebei, ${ }^{2}$ Department of Pharmacy, \\ Cangzhou Medical College, \\ Hebei, China
}

\begin{abstract}
Aim: To explore the expression and clinical significance of long noncoding RNA (lncRNA) gastric cancer-associated transcript 3 (GACAT3) in human colorectal cancer (CRC).

Methods: Expression of GACAT3 in CRC tissues and cell lines was measured using quantitative real-time PCR. CCK-8 and colony formation assays were used to assess the effect of GACAT3 on CRC cell line proliferation. Transwell invasion and migration assays were performed to detect the effect of GACAT3 on CRC cell line invasion and migration. Bioinformatics prediction, luciferase reporter assay, and pull-down assay were used to determine if miR-149 was a target of GACAT3. In addition, we also conducted colony formation assays and invasion assays to verify that GACAT3 promotes tumor progression through miR-149. Finally, in vivo tumorigenesis studies were used to demonstrate subcutaneous tumor growth.

Results: In the present study, we found that GACAT3 was highly expressed in CRC tissues and cell lines. Si-GACAT3 significantly decreased cell proliferation, motility, and invasiveness both in vitro and in vivo. We confirmed that downregulated GACAT3 significantly increased the expression of miR-149, and miR-149 binds to GACAT3 in a sequence-specific manner using luciferase reporter assays and pull-down assay. Further functional experiments indicated that GACAT3 could directly upregulate SP1 and STAT3 expressions by functioning as a competing endogenous RNA for miR-149, and consequentially, promoting CRC cell proliferation and invasion in vitro.
\end{abstract}

Conclusion: This study demonstrated that GACAT3 promotes tumor progression through competitive binding to miR-149 and suggests a promising new strategy for anti-CRC therapy.

Keywords: GACAT3, miR-149, colorectal cancer, growth, metastasis, SP1, STAT3

\section{Introduction}

Worldwide, colorectal cancer (CRC) is the third most common cancer and the fourth most common cause of cancer-related death. ${ }^{1}$ Despite the diagnosis and therapeutic advances in CRC, a significant proportion of CRC patients experience recurrence and die within 5 years after surgical treatment of their primary tumor. ${ }^{2}$ Thus, it is necessary to elucidate the molecular mechanisms underlying CRC proliferation. In the past decades, intensive investigations identified a variety of molecular markers for CRC characterization and prognosis. ${ }^{3,4}$ Recently, evidences have suggested that various long noncoding RNAs (lncRNAs) also act as modulators in carcinogenesis and progression of human CRC and may serve as novel therapeutic targets. ${ }^{5-7}$

As a novel class of regulatory genes, lncRNAs ( $>200$ nucleotides in length) have limited or no protein-coding capacity. ${ }^{8,9}$ Evidences suggest that lncRNAs play important
Correspondence: Lei Wang Department of Pharmacy, Cangzhou Medical College, Higher Education District, Cangzhou City, Hebei 06I00I, China Tel/fax +8603175306197 Email czmcwangleia@I26.com 
roles in a wide range of cellular processes, such as epithelialmesenchymal transition (EMT) ${ }^{10}$ and cell proliferation and migration. ${ }^{11}$

Studies indicate that lncRNAs are frequently aberrantly expressed in diverse human diseases, including various cancers. ${ }^{12,13}$ Several studies have revealed that lncRNAs can regulate gene expression at the transcriptional, posttranscriptional, and epigenetic levels. ${ }^{14,15} \mathrm{~A}$ large number of lncRNAs may function by competing with endogenous RNA (ceRNA) for miRNA targets, thereby preventing a single miRNA or multiple miRNAs from binding to their proper regulatory targets. ${ }^{16,17}$ A growing body of evidences strongly suggest that several lncRNAs, such as linc-NeD12,5, ${ }^{18}$ HOTAIR, ${ }^{19}$ and MALAT $1,{ }^{20}$ may function as ceRNAs, exerting essential roles in many biological processes.

Gastric cancer-associated transcript 3 (GACAT3), previously named as AC130710, has been officially named as GACAT3 now by the HUGO Gene Nomenclature Committee (HGNC). ${ }^{21}$ GACAT3 acts as a potential prognosis marker of GC. ${ }^{22}$ In GC cells, the expression of GACAT3 was suppressed by miR-129-5p, a tumor suppressor downregulated in the GC cells. ${ }^{23}$ However, the functions of GACAT3 in $\mathrm{CRC}$ and the underlying mechanisms remain unknown. In the present study, we found that GACAT3 was highly expressed in CRC. Knockdown of GACAT3 inhibited the malignancy of CRC cells, including proliferation, motility, and invasiveness. Meanwhile, we found that downregulated GACAT3 significantly increased the expression of miR-149. Bioinformatics, luciferase reporter assays, and pull-down assay confirmed that miR-149 binds to GACAT3 in a sequence-specific manner. This suggests that GACAT3mediated derepression of miR-149 is a potential mechanism by which this lncRNA promotes cell proliferation in CRC.

\section{Methods}

\section{Patient samples}

Twenty-six human CRC and their adjacent noncancerous tissues were collected from patients who underwent surgery for CRC at the Tumor Tissue Bank of Central Hospital of Cangzhou. There was no radiotherapy or chemotherapy prior to the operation. The adjacent noncancerous tissues were $5 \mathrm{~cm}$ from the edge of tumor. The diagnoses of these CRC and noncancerous tissues were verified by pathologists. All of the experimental protocols were conducted in accordance with the approved guidelines, and the study was approved by the Institutional Research Committee of Cangzhou Medical College. Written informed consent was provided by all the patients.

\section{Cell culture and transfection}

The following cell lines were obtained from the Cell Bank of the Chinese Academy of science: FHC, HT29, HCT116, SW480, LoVo, and 293T. Cells were cultured in Dulbecco's Modified Eagle's Medium, Iscove's modified Dulbecco's medium, or F-12K medium supplemented with $10 \%$ fetal bovine serum (FBS), $100 \mathrm{U} / \mathrm{mL}$ penicillin, and $100 \mathrm{mg} / \mathrm{mL}$ streptomycin (GIBCO, Carlsbad, CA, USA) in humidified air at $37^{\circ} \mathrm{C}$ with $5 \% \mathrm{CO}_{2}$. Cell transfections were performed using the Lipofectamine 3000 kit (Thermo Fisher Scientific, San Jose, CA, USA) according to the manufacturer's instructions.

\section{Vector construction}

GACAT3 gene inhibitor plasmid and the respective empty vector plasmids were purchased from GeneCopoeia ${ }^{\mathrm{TM}}$ (Rockville, MD, USA). The target sequence of si-GACAT3 is $5^{\prime}$-GGAUGAGAUGAUGAGGAUUTT-3'. The target GACAT3-wild and GACAT3-mutant gene expression plasmids (p-GACAT3'-UTR-wt and p-GACAT3' UTR-mut) were purchased from GeneCopoeia (Rockville, MD, USA). MiR-149 gene inhibitor plasmid was purchased from GeneCopoeia (Rockville, MD, USA) and its target sequence is 5'-AATTC AAAAAAGGGAGGGACGGGGGCTGT GC-3'.

\section{RNA extraction and quantitative real-time PCR analysis}

Samples were immediately frozen in liquid nitrogen after surgical dissection. Total RNA was extracted using TRIzol Reagent (Thermo Fisher Scientific, San Jose, CA, USA) according to the manufacturer's protocol. RNA quantity was measured by the Nano Drop ND-2000 spectrophotometer (optical density $260 \mathrm{~nm}$, Nano Drop, Wilmington, DE, USA), and RNA integrity was assessed using standard denaturing agarose gel electrophoresis. The expression of GACAT3 lncRNA from tissue samples or the cultured cells was quantified according to the manufacturer's instructions using SYBR Premix EX Taq ${ }^{\text {TM }}$ II kit (TaKaRa, RR820A) on the ABI Prism 7500 (Applied Biosystems, Foster City, CA, USA). MiRNA was harvested using the PureLink ${ }^{\mathrm{TM}}$ miRNA Isolation Kit (Thermo Fisher Scientific), and miRNA expression was quantified by TaqMan MicroRNA Assay Kit. The relative expression levels of mRNA/lncRNA and miRNA were determined using the $2^{-\triangle \Delta \mathrm{CT}}$ method with human glyceraldehyde 3-phosphate dehydrogenase (GAPDH) and U6 snRNA as internal controls, respectively. The sequences of the primers used in the study are shown in Table S1. Each experiment was performed in triplicate. 


\section{Western blot assay}

Tissue samples and the treated cells were lysed in Radio Immunoprecipitation Assay (RIPA) buffer (Thermo Scientific, Rockford, IL, USA) and Protease Inhibitor (Thermo Fisher Scientific, Waltham, MA, USA). The total protein $(30 \mu \mathrm{g})$ in equal concentration was separated by $10 \%$ SDS/PAGE gels and transferred to a polyvinylidene fluoride (PVDF) membrane (Millipore Corporation, Billerica, MA, USA). The PVDF membranes were blocked and incubated with the primary antibodies at $4{ }^{\circ} \mathrm{C}$ overnight. The membranes were then incubated with horseradish peroxidase-conjugated secondary antibody for $1 \mathrm{~h}$ at room temperature. All blots were visualized using an enhanced chemiluminescence (ECL) substrate kit (Amersham Biosciences, Little Chalfont, United Kingdom) and an ECL detection system (Amersham Biosciences). The antibodies used were rabbit anti-GAPDH (1:1,000; Santa Cruz Biotechnology, Santa Cruz, CA, USA) and rabbit anti-STAT3 (1:500; Abcam, Cambridge, UK).

\section{Bioinformatics prediction and luciferase reporter assay}

The potential miRNA binding sites of GACAT3 predicted by computer-aided algorithms were obtained from microRNA.org target program (www.microRNA.org). Two luciferase reporters containing the wild-type (WT) GACAT3 (psiCHECK2-GACAT3-WT) or mutant GACAT3 were generated to analyze the interaction between GACAT3 and miR-149. Mutant GACAT3 contained a mutation site (psiCHECK2-GACAT3-MU) abolishing targeting by miR-149. CRC cells were cotransfected with $500 \mathrm{ng}$ of the luciferase construct along with miR-149 and miR-149 control. At $48 \mathrm{~h}$ posttransfection, luciferase activity assays were performed with the Secrete-Pair ${ }^{\mathrm{TM}}$ Dual Luminescence Assay Kits (GeneCopoeia). The results were obtained from three independent experiments performed in duplicate.

\section{Pull-down assay with biotinylated DNA probe}

Pull-down assay was performed as reported. ${ }^{24,25}$ The GACAT3 pull-down probe sequence was $5^{\prime}$-AAGACTGA GGCCGACTGAGCCAGA-3', and the random pull-down probe sequence used as negative control was $5^{\prime}$-AAGACT GAGGCCGACTGCAAATCA-3'.

\section{Cell proliferation assays}

We used the Cell proliferation Reagent Kit I (3-(4,5dimethythiazol-2-yl)-2,5-diphenyl tetrazolium bromide [MTT]; Roche) to assess the ability of cell proliferation according to the manufacturer's instructions. The colony formation assay was performed as reported. ${ }^{26}$

\section{Cell migration and invasion assays}

In the migration assays, $1 \times 10^{4}$ cells at $48 \mathrm{~h}$ after transfection were placed into the upper chamber of an insert in serumfree medium (8- $\mu \mathrm{m}$ pore size, Millipore Corporation). For the invasion assays, $1 \times 10^{5}$ cells in serum-free medium were placed into the upper chamber coated with Matrigel (SigmaAldrich Co.). Medium containing 10\% FBS was added to the lower chamber. The cells remaining on the upper membrane were removed after incubation for $24 \mathrm{~h}$, and the cells that had migrated or invaded through the membrane were stained with $0.1 \%$ crystal violet, imaged, and counted using an IX71 inverted microscope (Olympus, Tokyo, Japan). All experiments were independently repeated three times.

\section{Animal studies}

For the subcutaneous xenograft model, $5 \times 10^{6}$ cells (stably transfected with $\mathrm{p}$-GACAT3-inhibitor and control vectors) were suspended in $100 \mu \mathrm{L}$ of PBS and then subcutaneously injected into the upper right flank region of each mouse. After 32 days, mice were sacrificed, and tumors were harvested and weighted. All of the experimental protocols were conducted in accordance with American Association for the Accreditation of Laboratory Animal Care guidelines and were approved by the Ethics Committee of Cangzhou Medical College.

\section{Statistical analysis}

All data were presented as the mean \pm SD and evaluated using SPSS 22 (SPSS Inc., Chicago, IL, USA). All statistical analyses were performed using ANOVA or a two-tailed Student's $t$-test to compare data. Differences were considered statistically significant at $P<0.05$.

\section{Results}

\section{Long noncoding RNA GACAT3 is highly expressed in CRC tissues and cell lines}

In order to investigate the role of GACAT3 in CRC tumorigenesis, we evaluated the expression of GACAT3 in 26 paired CRC tissues and adjacent normal tissues by quantitative realtime PCR (qRT-PCR). As shown in Figure 1A, compared with the adjacent normal mucosa tissues, the expression of GACAT3 was higher in 24 of 26 paired CRC specimens $(P<0.001)$. Meanwhile, we detected the expression of GACAT3 in the CRC cell lines HT29, HCT116, SW480, LoVo, and normal colorectal epithelium cell line FHC. 

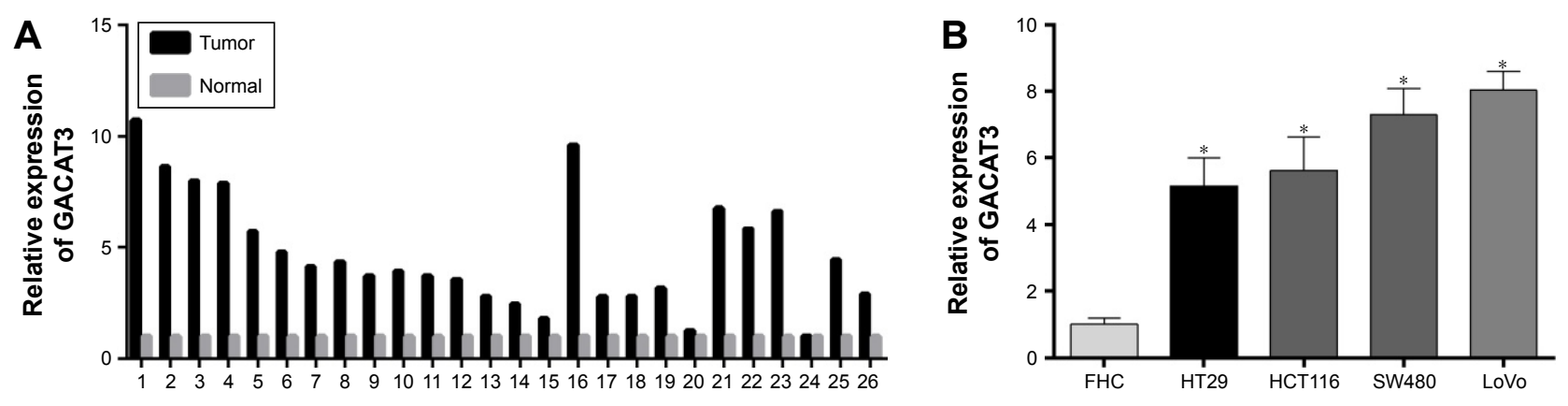

Figure I GACAT3 expression is elevated in CRC tissues and cell lines.

Notes: (A) The expression of GACAT3 was detected by qRT-PCR in 26 clinical CRC samples compared to those in adjacent normal tissue samples. (B) GACAT3 expression in four CRC cell lines HT29, HCTI 16, SW480, and LoVo was higher compared with the normal colorectal epithelium cell line FHC. $* P<0.05$.

Abbreviations: CRC, colorectal cancer; GACAT3, gastric cancer-associated transcript 3; qRT-PCR, quantitative real-time PCR.

The expression of GACAT3 was also significantly upregulated in four CRC cell lines compared with the normal colorectal epithelium cell line (Figure 1B).

\section{Knockdown of GACAT3 inhibited cell proliferation in vivo and in vitro}

To evaluate the roles of GACAT3 in CRC, we knocked down GACAT3 with si-GACAT3 in HT29 and LoVo cells and convinced that GACAT3 expression was significantly reduced in CRC cell lines by qRT-PCR (Figure 2A). The effect of GACAT3 on the proliferation of CRC cells was examined using the MTT assay. The results revealed that the ability of proliferation was suppressed with si-GACAT3 in both HT29 and LoVo cells (Figure 2B and C). Similarly, GACAT3 knockdown significantly decreased the clone formation activity in cell lines (Figure 2D and E).

To validate the function of GACAT3 in vivo, HT29/ sh-Con and HT29/sh-GACAT3 cells were subcutaneously injected into BALB/c-nu/nu mice. The effects of GACAT3 on tumor growth in nude mice were reflected by growth curves. The growth curves showed that GACAT3 promote the growth of tumor $(P<0.05$, Figure $2 \mathrm{~F})$. Also, the weight of tumors in the HT29/sh-Con group was obviously greater than that in the HT29/sh-GACAT3 group $(P<0.05$, Figure 2G).

\section{Knockdown of GACAT3 inhibited cell migration and invasion in vitro}

To investigate whether GACAT3 has a direct functional role in regulating the invasion and migration of CRC cells, we performed the invasion and migration assay. The results demonstrated that downregulation of GACAT3 significantly decreased the abilities of HT29 and LoVo cells to invade (Figure 3A and B). Similarly, migration of HT29 and LoVo cells was decreased following knockdown of GACAT3
(Figure 3C and D). These results indicate that GACAT3 could promote the migration and invasion of CRC cells.

\section{GACAT3 is a target of miR-I49}

Recent studies have suggested that lncRNA may function as a ceRNA or a molecular sponge in modulating the functions of miRNA. In order to screen out the miRNA associated with lncRNA, we performed the bioinformatics analysis, dualluciferase reporter assay, and pull-down assay.

First, the results of bioinformatics analysis (miRanda) of miRNA recognition sequences revealed that four miRNA binding sites were presented in GACAT3 cDNA, and they are miR-1207-5p, miR-149, miR-3649, and miR-3689b (Figure 4A). Meanwhile, we detected the expression of four potentially targeted miRNAs following the overexpression of GACAT3, and the results demonstrated that the expression level of miR-149 was significantly reduced and associated with the overexpression of GACAT3 (Figure 4B). Second, we found si-GACAT3 significantly increased the expression of miR-149 in HT29 and LoVo cells (Figure 4D, $P<0.05$ ). To investigate whether GACAT3 was a functional target of miR-149, we performed the dual-luciferase reporter assay. The results showed that the luciferase intensity of miR-149 was decreased in the GACAT3-Mut group $(P<0.05$; Figure $4 \mathrm{C}$ and E). Furthermore, we performed inverse pull-down assay to determine whether GACAT3 could target miR-149. As indicated in Figure 4F, miR-149 was precipitated by GACAT3 probe. Taken together, our results demonstrated that miR-149 could directly bind to GACAT3 at the miRNA recognition site.

\section{Knockdown of miR-I49 significantly reversed the inhibitory effects of si-GACAT3 on colorectal cells}

Although we have confirmed that GACAT3 is a target of miR-149, the role of miR-149 in GACAT3-induced 

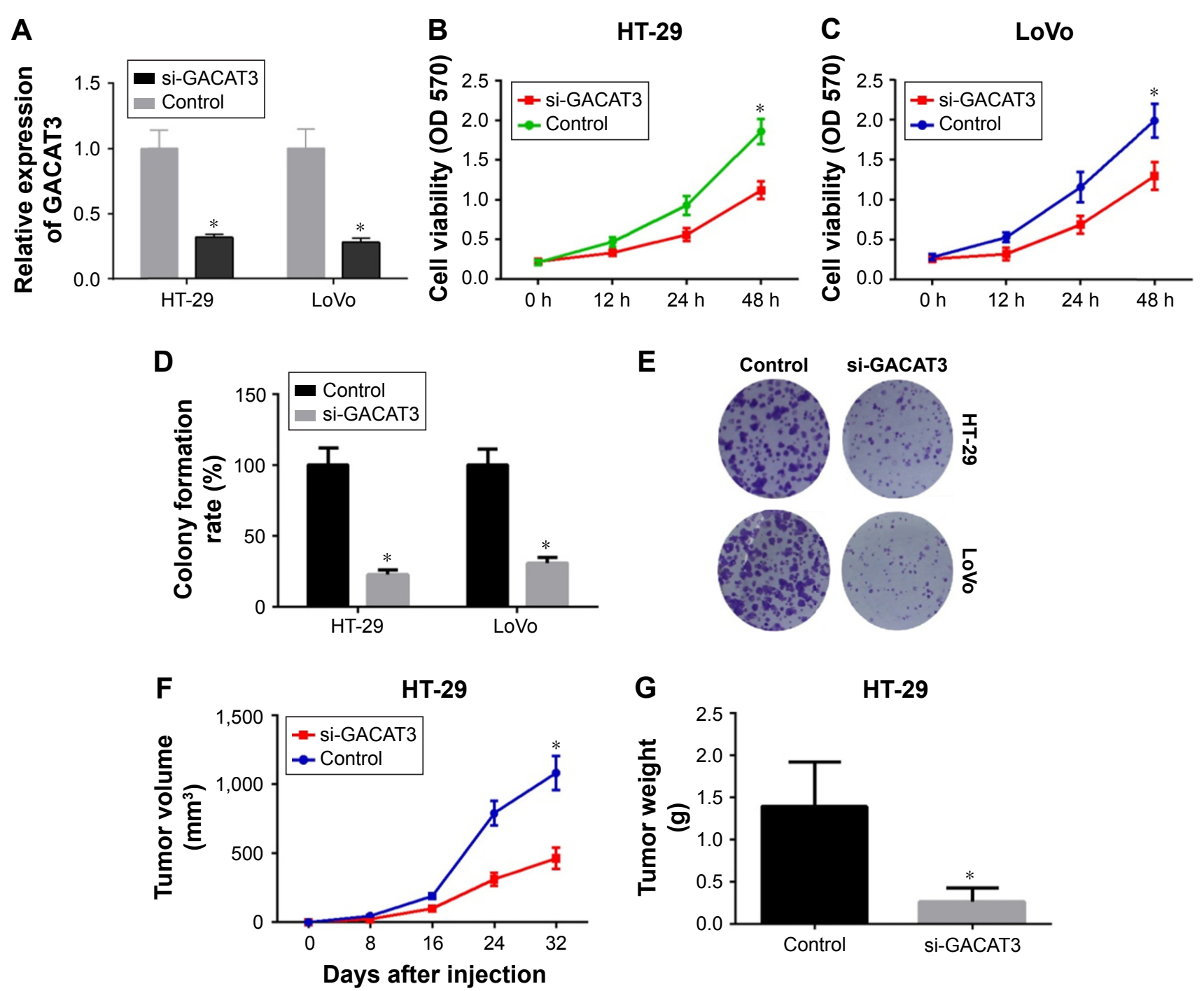

Figure 2 Knockdown of GACAT3 inhibited cell proliferation in vivo and in vitro.

Notes: (A) The si-GACAT3 or control was transfected into the CRC cell lines. GACAT3 levels were decreased significantly after transfection in HT-29 and LoVo cells. $* P<0.05$. (B, C) MTT assay was performed to determine the proliferation of HT29 and LoVo cells. GACAT3 silencing notably inhibited the cell proliferation. $* P<0.05$. (D, E) Colony formation ability was also inhibited by si-GACAT3 in HT29 and LoVo cells. $* P<0.05$. (F) Knockdown of GACAT3 in CRC cells significantly inhibits tumor growth in a mouse xenograft model, $* P<0.05$ compared with control. (G) Tumor weights of corresponding mouse xenograft models, $* P<0.05$ compared with control. Abbreviations: CRC, colorectal cancer; GACAT3, gastric cancer-associated transcript 3; MTT, 3-(4,5-dimethythiazol-2-yl)-2,5-diphenyl tetrazolium bromide.

promotion on colorectal cells remains unknown. As shown in Figure 5A, anti-miR-149, markedly downregulated miR-149 expression in HT29 and LoVo cells transfected with si-GACAT3 $(P<0.01)$. Figure 5B and $\mathrm{C}$ showed that knockdown of miR-149 in HT29 and LoVo cells, which stably transfected with si-GACAT3, significantly reversed si-GACAT3-induced inhibitory effects on cell clone formation and invasion. Furthermore, anti-miR-149 significantly increased the expression of SP1 inhibited by si-GACAT3 (Figure 5D). Meanwhile, Figure 5E and F showed that antimiR-149 significantly increased the expression of STAT3 inhibited by si-GACAT3. Altogether, our study demonstrated that GACAT3 promotes cell carcinogenesis of CRC through miR-149-mediated SP1 and STAT3 signaling (Figure 5G).

\section{Discussion}

Recently, many large-scale gene expression studies of tumor samples have revealed that many lncRNAs are misregulated in multiple cancers, ${ }^{27-29}$ including CRC ${ }^{30}$ For example, IncUBC1 is associated with poor prognosis and facilitates cell proliferation, migration, and invasion in CRC. ${ }^{31}$ Meanwhile, IncRNA NEAT1 impacts cell proliferation and apoptosis of CRC via regulation of Akt signaling. ${ }^{32}$ Moreover, the novel lncRNA TUSC7 could inhibit proliferation by sponging miR-211 in CRC. ${ }^{33}$ In this study, we found that IncRNA GACAT3 is significantly overexpressed in CRC tissues and correlated with the proliferation and invasion of $\mathrm{CRC}$ cells, indicating that GACAT3 plays an important role in CRC implicating the potential application of GACAT3 in the treatment of CRC. 
A

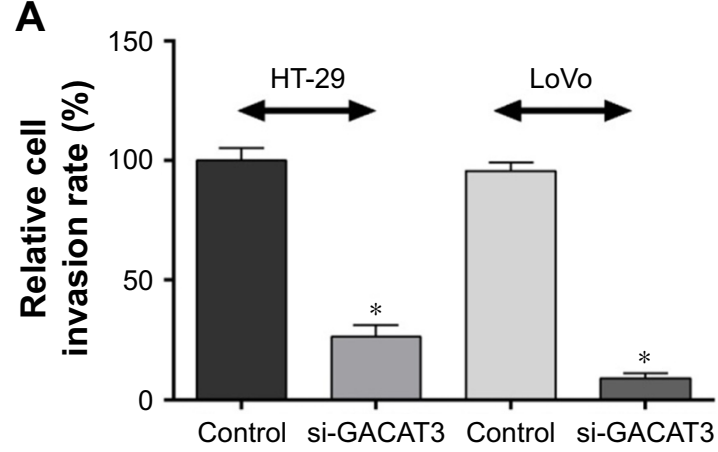

C

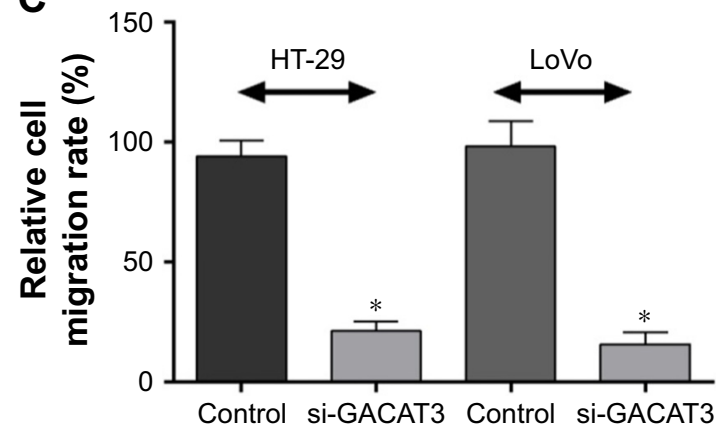

B

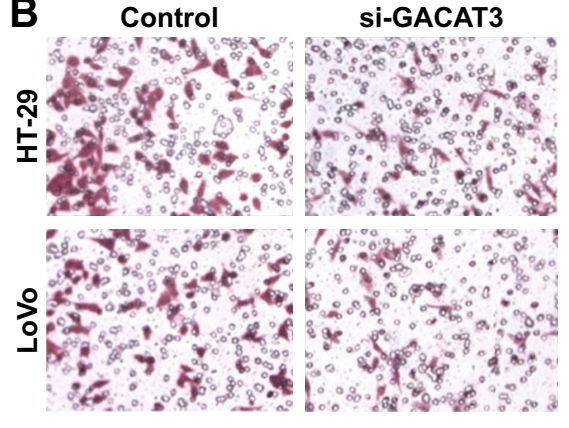

D Control

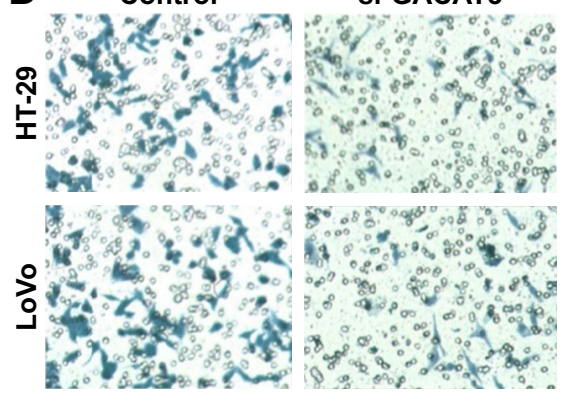

Figure 3 Knockdown of GACAT3 inhibited cell migration and invasion in vitro.

Notes: (A, B) Knockdown of GACAT3 decreased cell invasion by Transwell assay in HT-29 and LoVo cells. *P<0.05. (C, D) Knockdown of GACAT3 decreased cell migration by Transwell assay in HT-29 and LoVo cells. $* P<0.05$.

Abbreviation: GACAT3, gastric cancer-associated transcript 3.

A

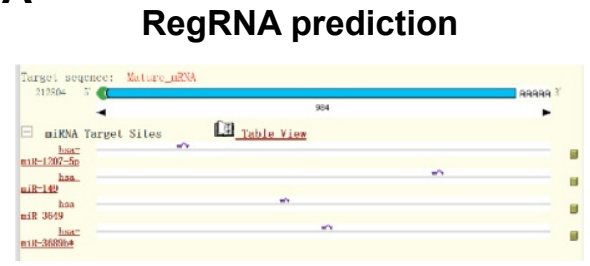

B

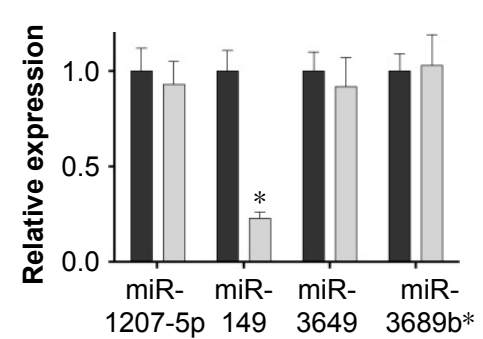

C

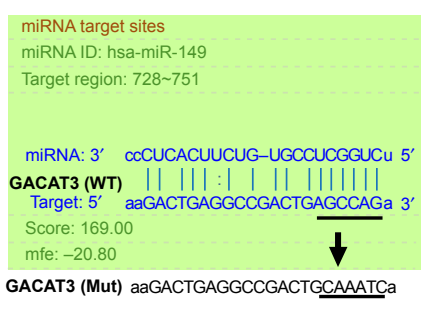

- Control $\square$ GCACT3
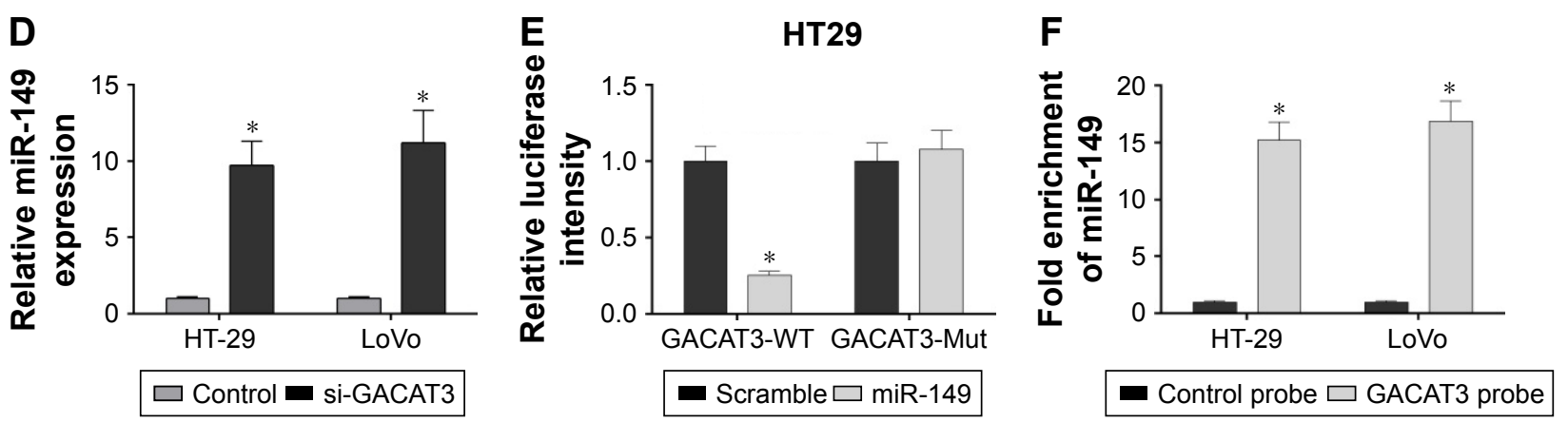

Figure 4 GACAT3 is identified as the functional downstream target of miR-149.

Notes: (A) The results of bioinformatics analysis showed the potentially targeted miRNA of GACAT3. (B) The expression of potentially targeted miRNA for GACAT3 was determined by qRT-PCR in 293 T cells. $* P<0.05$. (C) Sequence alignment of human GACAT3 with the miR-I49. The seed sequence of GACAT3 (middle) matches the 3'-UTR of miR-I49 (top). Bottom, mutation of the $3^{\prime}$-UTR of GACAT3 in the mutant luciferase reporter construct. (D) Application of si-GACAT3 increases miR- I49 levels in HT29 and LoVo cells. $* P<0.05$. (E) The luciferase reporter assay was used to detect the activity of GACAT3 in HT-29 cells cotransfected with IncRNA-CRNDE (Wt or Mut) and miR-149 mimics or scramble. ${ }^{*} P<0.05$. (F) The $q R T-P C R$ was performed to detect miR-149 in the samples pulled down by GACAT3 probe. $* P<0.05$.

Abbreviations: 3'-UTR, 3'-untranslated region; GACAT3, gastric cancer-associated transcript 3; qRT-PCR, quantitative real-time PCR. 
A

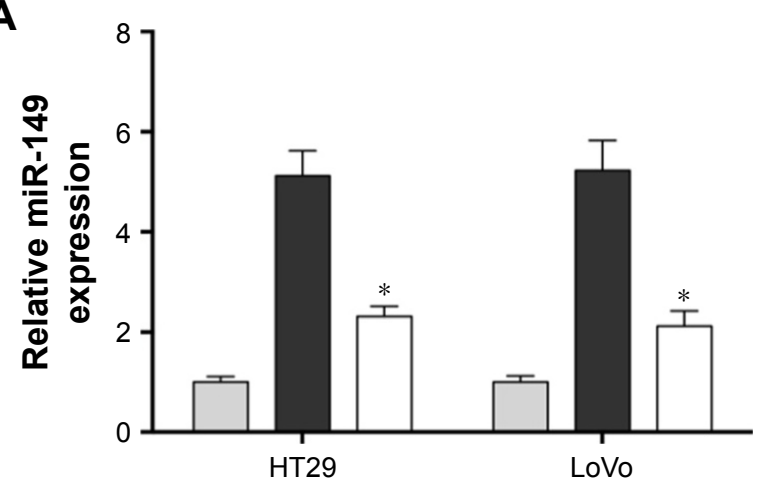

C

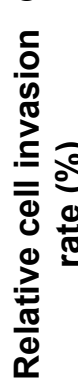

B

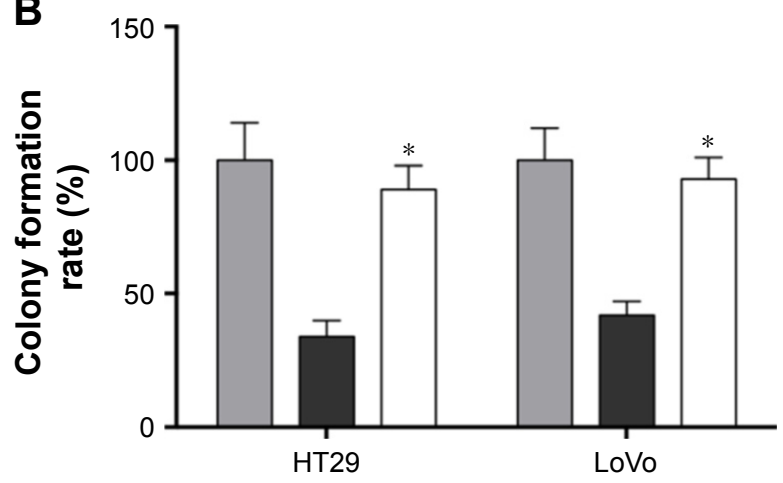

D

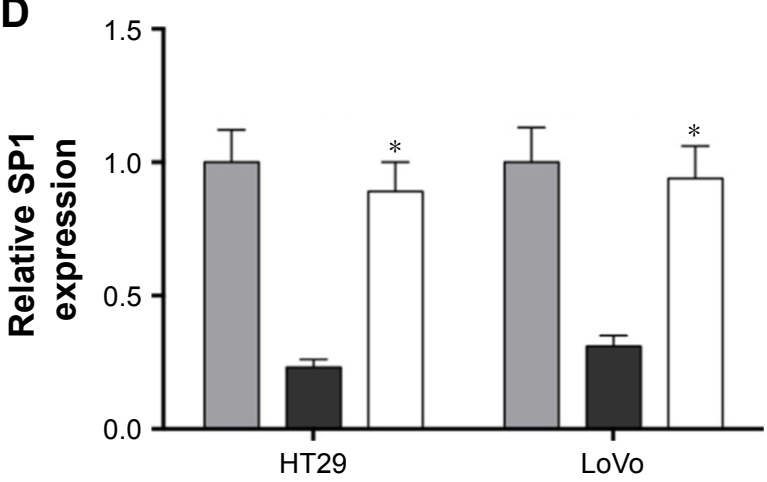

Control + anti-NC $\square$ si-GACAT3 + anti-NC $\square$ si-GACAT3 + anti-miR-149

$\mathbf{E}$

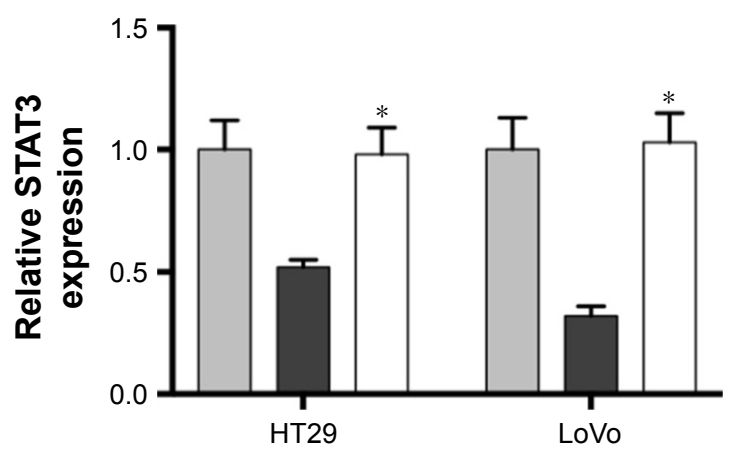

$\mathbf{F}$

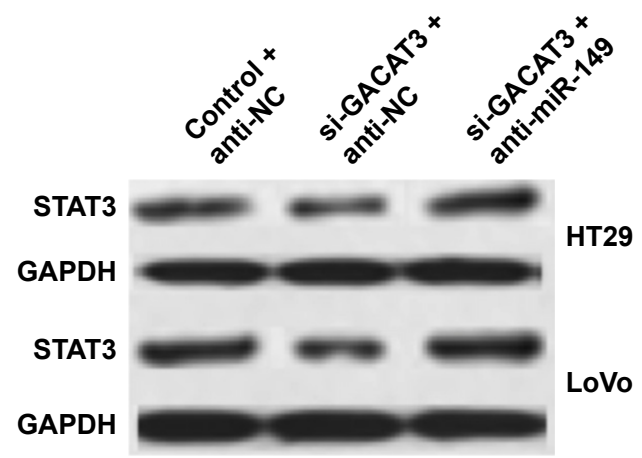

Control + anti-NC $\square$ si-GACAT3 + anti-NC si-GACAT3 + anti-miR-149

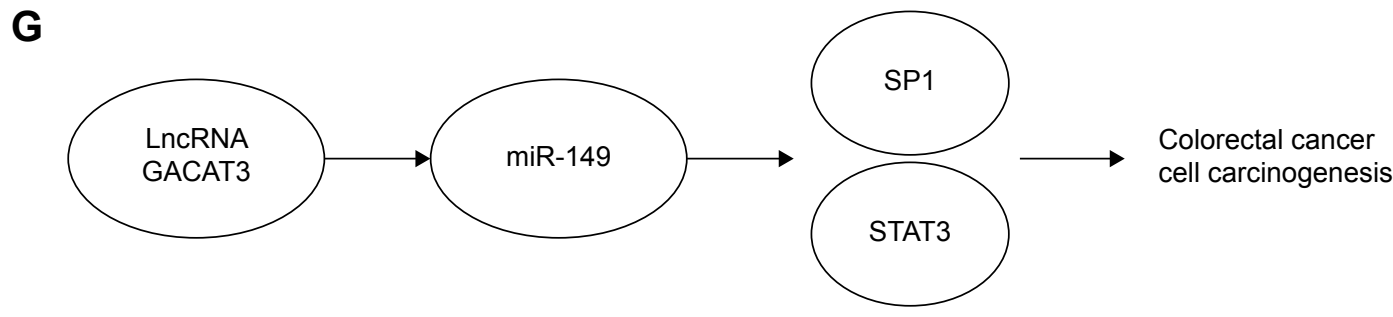

Figure 5 Regulation of CRC cell clone formation, invasion, and the expression of SPI and STAT3 by GACAT3 requires miR-I49.

Notes: (A) Expression levels of miR-149 were detected through qRT-PCR in HT29 and LoVo cells transfected with control, si-GACAT3, or anti-miR-I49. (B) Colony formation assay and invasion assay (C) were performed in HT29 and LoVo cells transfected with control, si-GACAT3, or anti-miR-I49. (D) Expression levels of SPI were determined in HT29 and LoVo cells transfected with control, si-GACAT3, or anti-miR-I49. (E, F) Expression levels of STAT3 were determined in HT29 and LoVo cells transfected with control, si-GACAT3, or anti-miR-I49. (G) Lnc GACAT3 promotes CRC cell carcinogenesis via miR-I49-mediated regulation of SPI and STAT3 signaling. *P $<0.05$. Abbreviations: CRC, colorectal cancer; GACAT3, gastric cancer-associated transcript 3; qRT-PCR, quantitative real-time PCR; SPI, specificity protein I. 
Previous researches have convinced that high expression of GACAT3 was first found in gastric cancer, ${ }^{22}$ and its expression level was significantly associated with poor prognosis. Overexpressed GACAT3 showed extensive potentials in cell proliferation, migration, and invasion through different mechanisms. For example, upregulated expression of GACAT3 enhanced cell proliferation in gastric cancer by inhibiting the relative expression of miR-129-5P. Besides, GACAT3 promotes gastric cancer cell proliferation through the IL-6/STAT3 signaling pathway. ${ }^{34}$ Similarly, in our study, highly expressed GACAT3 was found in CRC tissues and cell lines, as compared with adjacent histologically normal tissues and cell lines.

To verify the role of GACAT3 in CRC cell lines, HT29 and LoVo cells were transfected with GACAT3 siRNA and GACAT3 scramble, respectively. Downregulated GACAT3 was identified to suppress cell proliferation in HT29 and LoVo cells through MTT assay and the colony formation assay. The above results suggest that GACAT3 is higher expressed in CRC tissues and cell lines, and overexpressed GACAT3 promotes cell proliferation. Having identified that GACAT3 promoted the proliferation of CRC cells in vitro, we further explored the effect of GACAT3 in vivo. Downregulated expression of GACAT3 was found to significantly suppress tumor growth in si-GACAT3 model mice. The above results indicated that GACAT3 siRNA inhibits CRC growth in vivo. Meanwhile, Transwell assays were conducted to verify whether GACAT3 played an important role in migration and invasion of $\mathrm{CRC}$. The result suggested that both the migration and invasion abilities of CRC cells were largely suppressed by GACAT3 siRNA.

GACAT3 has been identified to possess oncogenic roles in tumor growth and metastasis, and it acts as a potential biomarker and therapeutic target in many types of human cancers. In accordance, overexpressed GACAT3 was detected in human CRC tissues and cell lines in this study. However, the underlying mechanism of GACAT3 in CRC is less well characterized. Thus, it is of a great significance to get a better understanding of the mechanism of GACAT3 in CRC. In this study, we revealed that GACAT3 regulates the growth and metastasis of CRC by targeting miR-149 for the first time.

Emerging evidence confirmed that lncRNA can act as endogenous miRNA sponges and competitively bind to miRNAs to regulate their expression..$^{35,36}$ To confirm whether GACAT3 serves as an miRNA sponge, we performed the bioinformatics analysis. The results showed that there was a binding site for miR-149 on GACAT3, and the expression of miR-149 was negatively correlated with GACAT3. Meanwhile, the level of miR-149 was upregulated by GACAT3 siRNA in HT29 and LoVo cells. Furthermore, luciferase activity assay showed that the intensity of fluorescence signal was largely weakened by miR-149 mimic in lncRNA GACAT3 WT group. Furthermore, the reverse pull-down assay verified that miR-149 can directly bind to GACAT3. Therefore, we are the first to prove that GACAT3 can directly be combined with miR-149.

Recently, an increasing number of researches revealed that miRNAs acted as tumor suppressors or oncogenes in many human cancers. ${ }^{37-39}$ Previous studies proved that miR-149 was downregulated in human cancers, ${ }^{40}$ and miR-149 inhibits non-small cell lung cancer cells EMT by targeting FOXM1. ${ }^{41}$ Other research showed that miR-149 inhibits proliferation and cell cycle progression through the targeting of ZBTB2 in human gastric cancer. ${ }^{42}$ Subsequently, miR-149 was identified to offset the role of GACAT3 on the viability and motility of CRC cells. Furthermore, we have detected the expression of specificity protein 1 (SP1, Sp1) as a target of miR-149. ${ }^{43}$ Meanwhile, we demonstrated that the expression of STAT3 inhibited by si-GACAT3 was reversed by anti-miR149. These above results indicate that GACAT3 regulates the proliferation and migration of CRC by targeting miR-149.

\section{Conclusion}

We found that GACAT3 was overexpressed in CRC tissues and cell lines. Downregulated GACAT3 was verified to suppress cell proliferation and migration in CRC cell lines. Further researches revealed that miR-149 was a direct target of GACAT3. Moreover, GACAT3 siRNA was verified to suppress CRC growth in vivo. Our research is the first to reveal the possible link between miR-149 and GACAT3 in CRC. The GACAT3-miR-149 pathway provides a new sight for CRC treatment.

\section{Acknowledgment}

This study was funded by the key project of Cangzhou Science and Technology Research and Development Program of China (No 162302105).

\section{Disclosure}

The authors report no conflicts of interest in this work.

\section{References}

1. Karsa LV, Lignini TA, Patnick J, Lambert R, Sauvaget C. The dimensions of the CRC problem. Best Pract Res Clin Gastroenterol. 2010; 24(4):381-396. 
2. Elias D, Faron M, Iuga BS, et al. Prognostic similarities and differences in optimally resected liver metastases and peritoneal metastases from colorectal cancers. Ann Surg. 2015;261(1):157-163.

3. Witalison EE, Cui X, Causey CP, Thompson PR, Hofseth LJ. Molecular targeting of protein arginine deiminases to suppress colitis and prevent colon cancer. Oncotarget. 2015;6(34):36053-36062.

4. Dickinson BT, Kisiel J, Ahlquist DA, Grady WM. Molecular markers for colorectal cancer screening. Gut. 2015;64(9):1485-1494.

5. Mercer TR, Dinger ME, Mattick JS. Long non-coding RNAs: insights into functions. Nat Rev Genet. 2009;10(3):155-159.

6. Ponting CP, Oliver PL, Reik W. Evolution and functions of long noncoding RNAs. Cell. 2009;136(4):629-641.

7. Nissan A, Stojadinovic A, Mitrani-Rosenbaum S, et al. Colon cancer associated transcript-1: a novel RNA expressed in malignant and premalignant human tissues. Int J Cancer. 2012;130(7):1598-1606.

8. Fatica A, Bozzoni I. Long non-coding RNAs: new players in cell differentiation and development. Nat Rev Genet. 2014;15(1):7-21.

9. St Laurent G, Wahlestedt C, Kapranov P. The landscape of long noncoding RNA classification. Trends Genet. 2015;31(5):239-251.

10. Shen F, Cai WS, Feng Z, et al. Long non-coding RNA SPRY4-IT1 promotes colorectal cancer metastasis by regulate epithelial-mesenchymal transition. Oncotarget. 2017;8(9):14479-14486.

11. Chen X, Liu B, Yang R, et al. Integrated analysis of long non-coding RNAs in human colorectal cancer. Oncotarget. 2016;7(17):23897-23908.

12. Zhu M, Liu JF, Xiao J, et al. Lnc-mg is a long non-coding RNA that promotes myogenesis. Nat Commun. 2017;8:14718.

13. Rao AKDM, Rajkumar T, Mani S. Perspectives of long non-coding RNAs in cancer. Mol Biol Rep. 2017;44(2):203-218.

14. Mercer TR, Mattick JS. Structure and function of long noncoding RNAs in epigenetic regulation. Nat Struct Mol Biol. 2013;20(3):300-307.

15. Yoon JH, Abdelmohsen K, Gorospe M. Posttranscriptional gene regulation by long noncoding RNA. J Mol Biol. 2013;425(19):3723-3730.

16. Wang K, Long B, Zhou LY, et al. CARL lncRNA inhibits anoxiainduced mitochondrial fission and apoptosis in cardiomyocytes by impairing miR-539-dependent PHB2 downregulation. Nat Commun. 2014;5:3596

17. Tay Y, Rinn J, Pandolfi PP. The multilayered complexity of ceRNA crosstalk and competition. Nature. 2014;505(7483):344-352.

18. Laneve P, Po A, Favia A, et al. The long noncoding RNA linc-NeD125 controls the expression of medulloblastoma driver genes by microRNA sponge activity. Oncotarget. 2017;8(19):31003-31015.

19. Cai H, Yao J, An Y, et al. LncRNA HOTAIR acts a competing endogenous RNA to control the expression of notch3 via sponging miR-613 in pancreatic cancer. Oncotarget. 2017;8(20):32905-32917.

20. Li C, Miao R, Liu S, et al. Down-regulation of miR-146b-5p by long noncoding RNA MALAT1 in hepatocellular carcinoma promotes cancer growth and metastasis. Oncotarget. 2017;8(17):28683-28695.

21. Chen SC, Li PF, Xiao BX, Guo JM. Long noncoding RNA HMlincRNA717 and AC1,30,710 have been officially named as gastric cancer associated transcript 2 (GACAT2) and GACAT3, respectively. Tumor Biol. 2014;35(1):8351-8352.

22. Xu C, Shao Y, Xia T, et al. IncRNA-AC130710 targeting by miR-129-5p is upregulated in gastric cancer and associates with poor prognosis. Tumor Biol. 2014;35(10):9701-9706.

23. Yu X, Song H, Xia T, et al. Growth inhibitory effects of three miR-129 family members on gastric cancer. Gene. 2013;532(1):87-93.

24. Wang P, Liu YH, Yao YL, et al. Long non-coding RNA CASC2 suppresses malignancy in human gliomas by miR-21. Cell Signal. 2015;27(2): 275-282.
25. Han P, Li JW, Zhang BM, et al. The lncRNA CRNDE promotes colorectal cancer cell proliferation and chemoresistance via miR-181a$5 \mathrm{p}$-mediated regulation of Wnt/beta-catenin signaling. Mol Cancer. 2017;16(1):9.

26. Yin DD, Liu ZJ, Zhang E, Kong R, Zhang ZH, Guo RH. Decreased expression of long noncoding RNA MEG3 affects cell proliferation and predicts a poor prognosis in patients with colorectal cancer. Tumour Biol. 2015;36(6):4851-4859.

27. Wang X, Sun W, Shen WF, et al. Long non-coding RNA DILC regulates liver cancer stem cells via IL-6/STAT3 axis. J Hepatol. 2016;64(1): 1283-1294.

28. Liu F, Yang F, Wu X, et al. Long non-coding RNA RBMY2FP promotes proliferation of male hepatocellular carcinoma by directing DNA methylation and activating RBMY1A1 via DNMT1. Oncotarget. 2016.

29. Jiang B, Sun R, Fang S, et al. Lnc-CC3 increases metastasis in cervical cancer by increasing Slug expression. Oncotarget. 2016;7(27): $41650-41661$

30. Su J, Zhang E, Han L, et al. Long noncoding RNA BLACAT1 indicates a poor prognosis of colorectal cancer and affects cell proliferation by epigenetically silencing of p15. Cell Death Dis. 2017;8(3):e2665.

31. Gao X, Wen J, Gao P, Zhang G, Zhang G. Overexpression of the long non-coding RNA, linc-UBC1, is associated with poor prognosis and facilitates cell proliferation, migration, and invasion in colorectal cancer. Onco Targets Ther. 2017;10:1017-1026.

32. Peng W, Wang Z, Fan H. LncRNA NEAT1 impacts cell proliferation and apoptosis of colorectal cancer via regulation of Akt signaling. Pathol Oncol Res. 2017;23(3):651-656.

33. Xu J, Zhang R, Zhao J. The novel long noncoding RNA TUSC7 inhibits proliferation by sponging MiR-211 in colorectal cancer. Cell Physiol Biochem. 2017;41(2):635-644.

34. Shen W, Yuan Y, Zhao M, et al. Novel long non-coding RNA GACAT3 promotes gastric cancer cell proliferation through the IL-6/STAT3 signaling pathway. Tumor Biol. 2016;37(11):14895-14902.

35. Huang G, Wu X, Li S, Xu X, Zhu H, Chen X. The long noncoding RNA CASC2 functions as a competing endogenous RNA by sponging miR-18a in colorectal cancer. Sci Rep. 2016;6:26524.

36. Liu F, Yuan JH, Huang JF, et al. Long noncoding RNA FTX inhibits hepatocellular carcinoma proliferation and metastasis by binding MCM2 and miR-374a. Oncogene. 2016;35(41):5422-5434.

37. Wang L, Bu P, Ai Y, et al. A long non-coding RNA targets microRNA miR-34a to regulate colon cancer stem cell asymmetric division. Elife. 2016;5:e14620.

38. Lin S, Gregory RI. MicroRNA biogenesis pathways in cancer. Nat Rev Cancer. 2015;15(6):321-333.

39. Garofalo M, Croce CM. Role of microRNAs in maintaining cancer stem cells. Adv Drug Deliv Rev. 2015;81:53-61.

40. Bischoff A, Huck B, Keller B, et al. miR149 functions as a tumor suppressor by controlling breast epithelial cell migration and invasion. Cancer Res. 2014;74(18):5256-5265.

41. Ke Y, Zhao W, Xiong J, Cao R. miR-149 inhibits non-small-cell lung cancer cells EMT by targeting FOXM1. Biochem Res Int. 2013; 2013:506731.

42. Wang Y, Zheng X, Zhang Z, et al. MicroRNA-149 inhibits proliferation and cell cycle progression through the targeting of ZBTB2 in human gastric cancer. PLoS One. 2012;7(10):e41693.

43. Wang F, Ma YL, Zhang P, et al. SP1 mediates the link between methylation of the tumour suppressor miR-149 and outcome in colorectal cancer. J Pathol. 2013;229(1):12-24. 


\section{Supplementary material}

Table SI The sequences of primer used for qRT-PCR

\begin{tabular}{|c|c|c|}
\hline$\overline{\text { Gene }}$ & Forward primer $\left(5^{\prime}-3^{\prime}\right)$ & Reverse primer $\left(5^{\prime}-3^{\prime}\right)$ \\
\hline GACAT3 & CTTCCGGAGCAGGTCTGAGT & CTTTCCCTGCAGAGACCAGT \\
\hline miR-I207-5p & GTTAGGGCAGGTGGGATG & TGTATGCGGCTGGTAAGTAG \\
\hline miR-I49 & GGCTCTGGCTCCGTGTCTT & CAGTGCAGGGTCCGAGGTATT \\
\hline $\operatorname{miR}-3649$ & ACACTCCAGCTGGGAGGGACCTGAGTG & TGGTGTCGTGGAGTCG \\
\hline$m i R-3689 b$ & TGCGGCTGGGAGGTGTGATAT & GTGCAGGGTCCGAGGT \\
\hline SPI & TGGCAGCAGTACCAATGGC & CCAGGTAGTCCTGTCAGAACTT \\
\hline GAPDH & GTCAACGGATTTGGTCTGTATT & AGTCTTCTGGGTGGCAGTGAT \\
\hline
\end{tabular}

Abbreviations: GACAT3, gastric cancer-associated transcript 3; GAPDH, glyceraldehyde 3-phosphate dehydrogenase; qRT-PCR, quantitative real-time PCR; SPI, specificity protein I.

\section{Publish your work in this journal}

OncoTargets and Therapy is an international, peer-reviewed, open access journal focusing on the pathological basis of all cancers, potential targets for therapy and treatment protocols employed to improve the management of cancer patients. The journal also focuses on the impact of management programs and new therapeutic agents and protocols on patient perspectives such as quality of life, adherence and satisfaction. The manuscript management system is completely online and includes a very quick and fair peer-review system, which is all easy to use. Visit http://www.dovepress.com/testimonials.php to read real quotes from published authors. 